\title{
Erratum to: Hadronic shift in pionic hydrogen
}

\author{
M. Hennebach ${ }^{1, a}$, D.F. Anagnostopoulos ${ }^{2}$, A. Dax ${ }^{3}$, H. Fuhrmann ${ }^{4}$, D. Gotta ${ }^{1, b}$, A. Gruber ${ }^{4, c}$, A. Hirtl $^{4, c}$, \\ P. Indelicato ${ }^{5,6,7}$, Y.-W. Liu ${ }^{3, d}$, B. Manil ${ }^{5, \text { e }}$, V.E. Markushin ${ }^{3, f}$, A.J. Rusi el Hassani ${ }^{8}$, L.M. Simons ${ }^{3}$, \\ M. Trassinelli ${ }^{9,10}$, and J. Zmeskal ${ }^{4}$ \\ 1 Institut für Kernphysik, Forschungszentrum Jülich, D-52425 Jülich, Germany \\ 2 Department of Materials Science and Engineering, University of Ioannina, GR-45110 Ioannina, Greece \\ 3 Laboratory for Particle Physics, Paul Scherrer Institut, CH-5232 Villigen, Switzerland \\ 4 Stefan Meyer Institute for Subatomic Physics, Austrian Academy of Sciences, A-1090 Vienna, Austria \\ ${ }^{5}$ Laboratoire Kastler Brossel, Sorbonne Universités, UPMC Univ. Paris 06, Case 74; 4, place Jussieu, 75005 Paris, France \\ 6 Laboratoire Kastler Brossel, CNRS, 75005, Paris, France \\ 7 Laboratoire Kastler Brossel, Département de Physique de l'École Normale Supérieure, 24 Rue Lhomond, 75005, Paris, France \\ 8 Faculté des Sciences et Techniques, Université Abdelmalek Essaâdi, Tanger, Morocco \\ 9 Institut des NanoSciences de Paris, Sorbonne Universités, UPMC Univ. Paris 06, Case 840; 4, place Jussieu, Paris, France \\ 10 Institut des NanoSciences de Paris, CNRS, F-75005, Paris, France
}

Original article: Eur. Phys. J. A (2014) 50: 190, https://doi.org/10.1140/epja/i2014-14190-x

Received: 16 January 2019

Published online: 21 February 2019

(C) Società Italiana di Fisica / Springer-Verlag GmbH Germany, part of Springer Nature, 2019

The numerical value given in sect. 6.2 for the sum of the QCD quantity $a^{+}+a^{-}$is incorrect. The correct version of eq. (9) in sect. 6.2 is

$$
a^{+}+a^{-}=(93.0 \pm 3.3) \cdot 10^{-3} m_{\pi}^{-1} .
$$

Publisher's Note The EPJ Publishers remain neutral with regard to jurisdictional claims in published maps and institutional affiliations.

\footnotetext{
a Present address: Areva GmbH, D-63067 Offenbach, Germany.

b e-mail: d.gotta@fz-juelich.de (corresponding author)

c Present address: Universitätsklinik für Radiologie und Nuklearmedizin, Medizinische Universität Wien, A-1090 Vienna, Austria.

d Present address: Physics Department, National Tsing Hua University, Hsinchu 300, Taiwan.

e Present address: Laboratoire de Physique des Lasers, CNRS et Université Paris 13, France.

f Present address: Department of IT, Paul Scherrer Institut, CH-5232 Villigen, Switzerland.
} 